\title{
Stand-to-Sit Maneuver in Paraplegia after Spinal Cord Injury using Functional Neuromuscular Stimulation
}

\author{
Sarah R. Chang, Rudi Kobetic, Member, IEEE and Ronald J. Triolo, Member, IEEE
}

\begin{abstract}
The paralysis resulting from spinal cord injury (SCI) can compromise the independent performance of many activities of daily living. Neuroprostheses utilizing functional neuromuscular stimulation (FNS) are able to restore standing and stepping after SCI by actively contracting the paralyzed muscles. The stand-to-sit maneuver, however, requires good control of eccentric contractions of the knee and hip musculature, which is difficult to achieve with FNS alone. This preliminary study compares the biomechanics of the stand-to-sit maneuver with open-loop FNS to able-bodied in terms of upper extremity forces and sitting impact force. With FNS, the upper extremity forces reached an average of $25 \%$ of their body weight more than able-bodied controls during the maneuver. Individuals with SCI also exhibited twice the impact force when making contact with the chair as compared to able-bodied subjects. These large forces and quick descents are potential for injury and an improved method for controlling the stand-to-sit maneuver when using FNS is necessary.
\end{abstract}

\section{INTRODUCTION}

There are approximately 270,000 people in the United States living with spinal cord injury (SCI), where $43 \%$ have paraplegia [1]. The paralysis resulting from SCI can compromise daily function and severely impair the ability to stand and complete simple mobility maneuvers. Up to $38 \%$ of persons with paraplegia rank walking as a high priority to improve their quality of life [1]. Prerequisites to walking include standing up and sitting down. Functional neuromuscular stimulation (FNS) and passive or powered external bracing are two established methods for individuals with SCI to accomplish sit-to-stand, standing or stepping, and stand-to-sit maneuvers.

Eccentric contractions or lengthening of the active quadriceps muscles are essential for stand-to-sit maneuvers. However, eccentric contractions are not well controlled when using FNS due to a lack of feedback to appropriately

This work was supported by The Rehabilitation Research \& Development Service of Veterans Affairs Grant B0608-R. S.R. Chang was also supported in part by GAANN P200A100112 to Case Western Reserve University, Biomedical Engineering.

S. R. Chang is with the Department of Biomedical Engineering, Case Western Reserve University, Cleveland, OH 44106 USA, and also with the Louis Stokes Cleveland Department of Veterans Affairs Medical Center, Cleveland, OH 44106 (e-mail: sarah.r.chang@case.edu).

R. Kobetic is with the Motion Study Laboratory, Louis Stokes Cleveland Department of Veterans Affairs Medical Center, Cleveland, OH 44106 USA (e-mail: rkobetic@fescenter.org).

R. J. Triolo is with the Department of Orthopaedics, Case Western Reserve University, Cleveland, OH 44106 USA, and also with the Louis Stokes Cleveland Department of Veterans Affairs Medical Center, Cleveland, OH 44106 (e-mail: ronald.triolo@case.edu). modulate the extensor muscles as the body's center of mass moves throughout the maneuver [2].

Control of the stand-to-sit maneuver in persons with SCI is important for protecting the insensate tissues of the buttocks, especially when sitting down on a hard surface. As Dolan et al. found in their study, the subject was essentially sitting down without the use of the stimulation because she only started to descend once the stimulation terminated [3]. Sitting down without any control may cause a high sitting impact when first making contact with the chair. This can be dangerous and potentially cause deep tissue injury, which is typically not visible to the naked eye or can appear as bruising. Deep tissue injury occurs when underlying soft tissues are damaged from pressure or shear, either from a sudden high impact force or from repeated forces over an extended period of time. In particular, the ischial tuberosity is the bony prominence that impacts the gluteus muscles and nearby blood vessels when sitting down. This injury can be even more extreme for people with SCI whose gluteus muscles may be atrophied and unable to withstand the impact that may occur in the stand-to-sit maneuver.

Closed-loop controllers have been developed for standing up and sitting down with FNS that attempt to address these issues. Dolan et al. designed a switching curve controller for people with paraplegia who utilize FNS that alternated the stimulation between on and off based on the knee angle position and knee angular velocity in relation to a switching curve. The switching curve controller was able to reduce the knee angular velocities and accelerations of the subject using stimulation, but the terminal angular velocities and angular accelerations were still at least twice as high as those for able-bodied [3]. Poboroniuc et al. created a new control strategy, called ONZOFF, to assist FNS driven sitting down that improved upon the switching curve controller concept to include a zone between on and off that gradually changed the levels of stimulation. This ONZOFF controller was able to significantly lower the knee end velocity by approximately half when compared to the On/Off controller. The hand forces supporting the body weight on a walker using the ONZOFF were approximately $50 \%$ of the subject's body weight. It was also found that there was difficulty in selecting the correct switching line for the On/Off controller due to perturbations and inconsistencies with the arm supports [4].

This paper reports preliminary data for understanding the biomechanics during able-bodied stand to sit and sitting down with open loop FNS that is necessary to refine these closed-loop approaches or define new and more effective interventions that may include a combination of stimulation and external bracing to safely accomplish the maneuver. 


\section{METHODS}

\section{A. Subjects}

The stand-to-sit maneuver was evaluated in two subjects with paraplegia using open-loop FNS to complete the maneuver (Subject A: ASIA A, T7 Injury Level, 51 years old, $67 \mathrm{~kg}, 174 \mathrm{~cm}$ and Subject B: ASIA C (MMT - hip extensors Grade 1 and knee extensors Grade 2), T6 Injury Level, 47 years old, $58 \mathrm{~kg}, 168 \mathrm{~cm}$ ). Two able-bodied subjects were used as controls (Subject C: 23 years old, 80 $\mathrm{kg}, 180 \mathrm{~cm}$ and Subject D: 25 years old, $80 \mathrm{~kg}, 185 \mathrm{~cm}$ ). All subjects in this study signed a consent form approved by an institutional review board prior to participation.

\section{B. Equipment and Setup}

During the experiments, all subjects held onto a walker instrumented with six-axis load cells (AMTI, Watertown, MA, USA) under each hand to measure the upper extremity (UE) forces occurring throughout the maneuver. The subjects sat down onto a chair covered with a $13 \mathrm{~mm}$ thick rubber foam and instrumented with a force plate (AMTI, Watertown, MA, USA) that measured the sitting impact force when the subjects first made contact with the chair. To account for the entire body weight, all subjects stood with each foot on a separate force plate (AMTI, Watertown, MA, USA). Forces were collected at $1000 \mathrm{~Hz}$. Reflective markers were placed bilaterally on the upper body (acromion process, lateral epicondyle of the elbow, wrist, and third metacarpal), the trunk and pelvis (C7, T6, anterior and posterior superior iliac spines, and sacrum), and the lower extremities (trochanter, lateral surface of mid-thigh, lateral epicondyle of the knee, lateral surface of mid-shank, lateral malleolus, calcaneous, and second metatarsal head). A Vicon MX40 motion capture system (Vicon Motion Systems, Oxford, UK) was utilized to record kinematic data (i.e. marker trajectories) at $200 \mathrm{~Hz}$, and this information was used to determine kinematics and kinetics of the stand-to-sit maneuver in post-processing.

\section{Trials Performed}

Sitting down with FNS was accomplished with a customized pre-programmed pattern that ramped down the stimulation applied bilaterally to the hip and knee extensor muscles optimized by a physical therapist for each subject. This ramp down pattern occurred over the length of two seconds. For Subject A, the hip extensors used include the posterior portion of the adductor magnus and gluteus maximus, and the knee extensors used include the vastus lateralis and vastus intermedius. For Subject B, the hip extensors included the posterior portion of the adductor magnus, hamstrings, and gluteus maximus, and the knee extensors used include vastus lateralis and vastus intermedius. Able-bodied participants were asked to perform the stand-to-sit maneuver at a comfortable pace. All subjects performed at least five repetitions of the stand-to-sit maneuver. The trial began in a standing position and ended in a seated position on the fixed, instrumented chair with hands on the instrumented walker. Subjects with SCI stood up with FNS and assumed a comfortable standing position before initiating the maneuver. The chair was set to a standard height of $48 \mathrm{~cm}$, the average chair height based on popliteal height [5]. For each trial, marker trajectories, force plate data, and load cell data were collected.

\section{Data Processing}

All data was processed offline. The Vicon Nexus software was used to label and process the marker trajectories. A $5^{\text {th }}$ order low pass Butterworth filter with a cutoff frequency of $10 \mathrm{~Hz}$ was applied to the kinematic and analog signals to filter and smooth the data, respectively. Ensemble averages were calculated using five trials for each of the outcome measures.

The length of the trial for the subjects with paraplegia was determined by the start and end of the stimulation pattern. If the subject did not make contact with the chair when the stimulation ended, the trial end was determined by the peak initial sitting impact force on the chair. The length of the trial for the able-bodied participants was determined by a deviation of three degrees with respect to the stationary initial and final knee joint angles [6].

The right and left vertical component of the upper extremity forces were averaged together and normalized by the subject's body weight $(\mathrm{BW})$. The sitting impact force was the peak force that occurred just after the subject made contact with the instrumented chair and was normalized by the subject's body weight.

Minitab 16 Statistical Software was used to determine whether the data had statistically significant differences. A one-way analysis of variance (ANOVA) with a $95 \%$ confidence interval $(\mathrm{p}<0.05)$ was performed to determine statistical significance between subjects using FNS and ablebodied controls.

\section{RESULTS}

The knee angle during the maneuver ranged between $0^{\circ}$ (standing position) and $89.0 \pm 14.2^{\circ}$ (sitting position) for ablebodied and $0^{\circ}$ (standing position) and 82.5 $\pm 9.9^{\circ}$ (sitting position) for subjects using FNS, as shown in Figures 1 and 2 respectively. Differences in ending knee angle during sitting were influenced by positioning of the feet and the subjects' final sitting location on the chair. The hip angle ranged between $0^{\circ}$ and $75.0 \pm 8.8^{\circ}$ for able-bodied (Figure 1) and $0^{\circ}$ and $65.0 \pm 11.1^{\circ}$ for subjects using FNS (Figure 2). Knee angular velocity for able-bodied and FNS users during the maneuver reached different maximum values. Ablebodied subjects' maximum flexion knee angular velocity was $78.9 \pm 20.3 \%$ (Figure 1), and subjects using FNS had maximum flexion knee angular velocities of $203.3 \pm 75.8 \%$ (Figure 2). Subjects using FNS had a significantly higher $(\mathrm{p}=0.001)$ maximum flexion knee angular velocity than that of able-bodied individuals.

The total vertical component of the upper extremity forces for the subjects with SCI (Figure 3) reached a maximum during the stand-to-sit maneuver that was an average of $25 \%$ of body weight (Subject A: $25.0 \pm 5.7 \% \mathrm{BW}$ and Subject B: $26.9 \pm 2.8 \% \mathrm{BW}$ ) as compared to the approximately $5 \%$ body weight for able-bodied subjects (Subject C: $4.0 \pm 1.6 \% \mathrm{BW}$ and Subject D: $6.3 \pm 0.6 \% \mathrm{BW}$ ). 


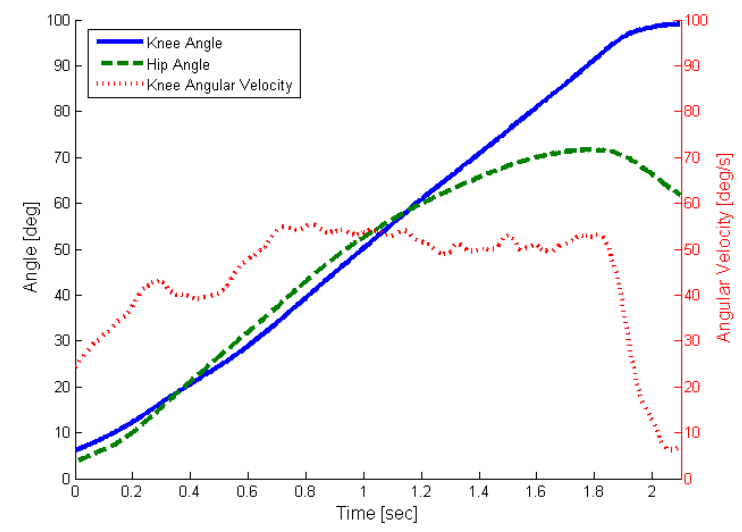

Figure 1. Typical plot of the knee angle, hip angle, and knee angular velocity for able-bodied subjects.

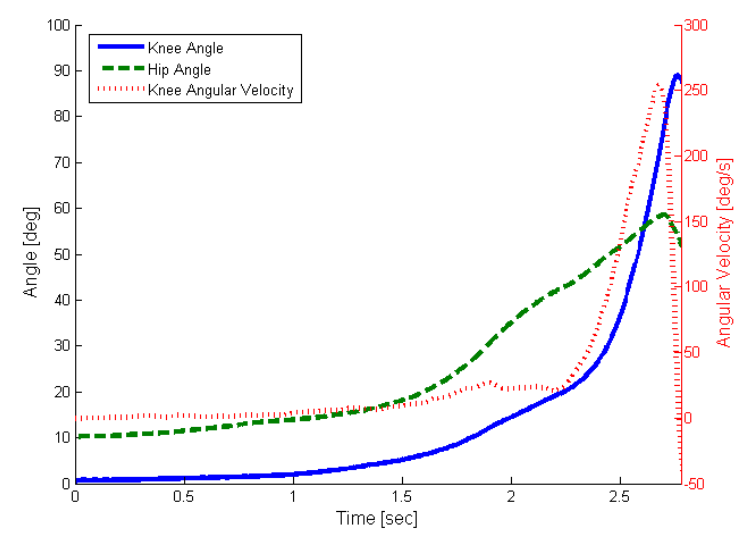

Figure 2. Typical plot of the knee angle, hip angle, and knee angular velocity for subjects with SCI using FNS.

No significant difference in upper extremity loading was observed between SCI subjects ( $\mathrm{p}=0.338$ ), while they put significantly more support on the walker than able-bodied subjects $(\mathrm{p}<0.001)$.

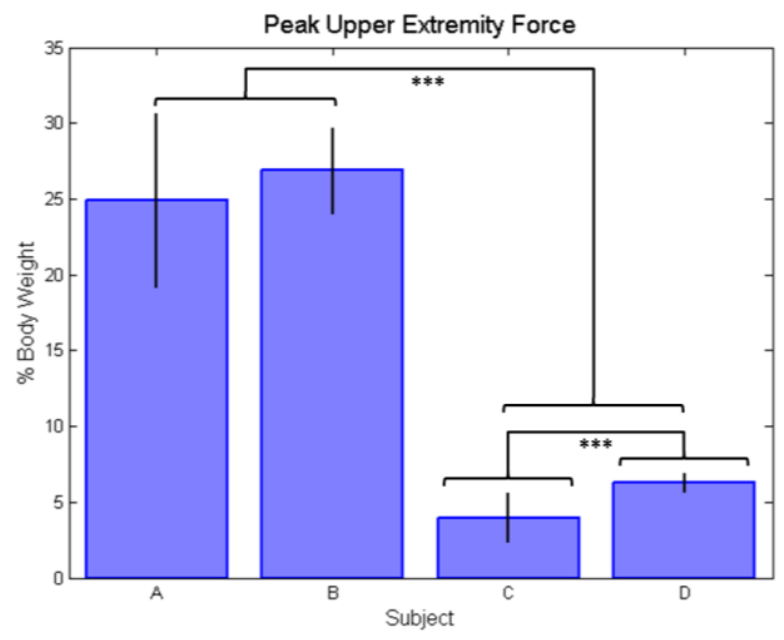

Figure 3. Means and standard deviations for peak upper extremity forces for able-bodied and subjects using FNS $* * *=(\mathrm{p}<0.05)$

The impact forces, shown in Figure 4, for subjects using FNS were approximately 1.3-2 times their own body weight
(Subject A: $173.7 \pm 26.6 \%$ BW and Subject B: $165.5 \pm 38.9$ $\% \mathrm{BW}$ ) and twice as high as able-bodied subjects which was approximately 0.7 times body weight (Subject C: $79.6 \pm 6.9$ \%BW and Subject D: $62.9 \pm 4.7 \% \mathrm{BW})$. The peak impact force was not significantly different between the subjects using FNS ( $p=0.709)$, while they generated a significantly larger impact force than the able-bodied subjects $(\mathrm{p}=0.002)$.

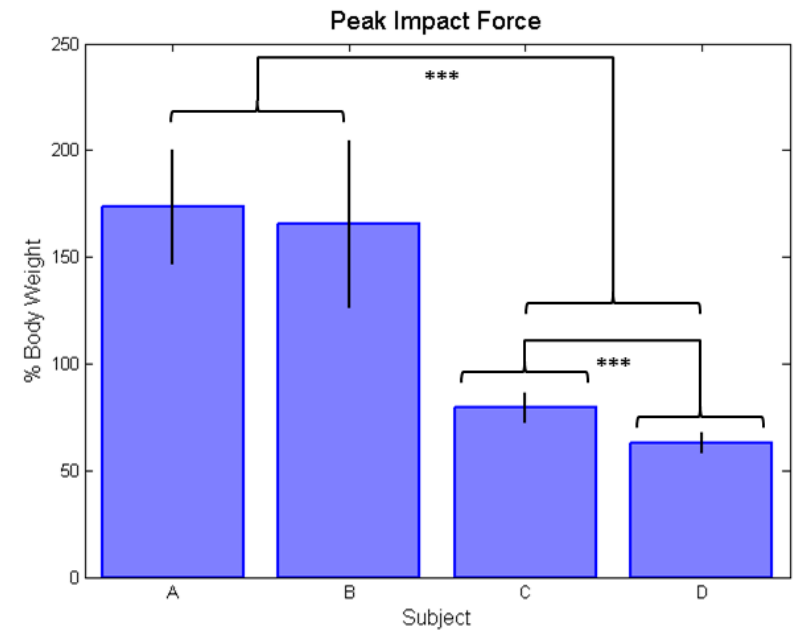

Figure 4. Means and standard deviations for peak impact force for able-bodied and subjects using FNS. $* * *=(p<0.05)$

To summarize, the upper extremity and impact forces were not significantly different within the two subjects with SCI. The peak UE loads and impact forces were found to be significantly different between the two able-bodied subjects. Both upper extremity and impact force parameters were found to be significantly higher for SCI subjects when compared to able-bodied controls.

\section{DISCUSSION}

The stand-to-sit maneuver is currently performed using an open-loop pattern of stimulation that ramps down at a constant rate. This results in high UE loads and impact forces in excess of those observed for able-bodied sitting. FNS users heavily relied on their upper extremities to support their body weight and decelerate the body during descent in an attempt to reduce the sitting impact force with the chair. The UE forces for subjects with SCI reach a maximum during the stand-to-sit maneuver that is approximately 5 times larger than that for able-bodied controls. Able-bodied individuals do not typically use their upper extremities when sitting down. Subjects were not required to use their arms and could use the walker as desired. However, they were instructed to have their hands on the walker at all times during the maneuver. These UE forces for Subjects C and D merely measured the weight of their arms resting on the walker and do not indicate any reliance on their upper extremities to complete the maneuver. The slight increase in upper extremity force for Subject D is reflected in this subject's reduced impact force. The UE forces in individuals with SCI were comparable or less than those reported for some closed-loop controllers [4].

The able-bodied individuals were capable of controlling their stand-to-sit maneuver through eccentric contractions of 
the quadriceps and coordination of hip and knee angles throughout the maneuver. As a result, the able-bodied impact force was measured to be less than their body weight. The difference in impact force between the able-bodied subjects may be due to subject preference on a comfortable pace for the maneuver. If the subject decided to descend at a slower rate than the other subject, the impact force would be smaller. Since the subjects' feet remained on the floor throughout the maneuver, some of the body weight was transmitted through the legs and the final weight measured by the instrumented chair was less than body weight. The forces on the feet, or the ground reaction force, for ablebodied subjects were generally symmetrical between the right and left legs. The force tended to be slightly greater on one leg than the other, which may be due to the natural tendency to lean more towards one leg or to periodically shift weight between the right and left sides. Subjects using FNS, on the other hand, showed more asymmetry between the right and left ground reaction forces. Asymmetry in the ground reaction forces may be influenced by the inability to physically sense how the weight is distributed and the varying strength of the muscle contractions generated by the FNS controlling motion in the frontal plane. Despite the asymmetry at the legs, their upper extremity forces were generally symmetrical throughout the maneuver.

In general, these results were somewhat expected. Visual observation of the stand-to-sit maneuver for people using FNS reveals that an increased impact occurs when making contact with the seat. However, the magnitude of the impact was unknown. The knee angular velocity was comparable to values found in previous studies of the stand-to-sit maneuver [3]. However, the knees remained locked during most of the maneuver, even as stimulation to the quadriceps was decreasing, until a critical value was reached after which the knees flexed rapidly and subjects were in near free-fall. Hip flexion angle followed similar trends. Subjects with SCI adapted various strategies in addition to upper extremity effort to minimize impact force and control knee angular velocity, such as leaning further forward at the hips and trunk. If the subjects' knee angular velocity can be reduced and better controlled by either modulating stimulation differently or damping knee flexion with an appropriately tuned orthosis, then it should be possible to reduce the impact force and increase safety.

The limitations of this study involve the age and number of subjects. There is a significant difference in age of the able-bodied controls and subjects using FNS. It is assumed that the able-bodied subjects are representative of the total population. Moreover, these results were determined based on a small number of subjects. Since this study is in its preliminary stages, further experiments are in the process of being performed with additional subjects.

Although the parameters appear to be of the same magnitude as those reported for closed-loop controllers, there is room for significant improvement which should reduce the UE effort and high impact forces that have the potential to lead to soft-tissue injury. The results of these preliminary data call for a better intervention to allow controlled knee flexion that utilizes the benefits of FNS to contract the paralyzed muscles and simultaneously provide sufficient body support as the user descends to the chair. Implementation of an external damping system in combination with closed-loop FNS may provide the capability to improve the control of the stand-to-sit maneuver.

\section{ACKNOWLEDGMENT}

The authors would like to thank the subjects for their participation in the study and Lisa Lombardo, PT for her expertise and assistance in preparation for and during the experiments.

\section{REFERENCES}

[1] K. D. Anderson, "Targeting Recovery: Priorities of the Spinal CordInjured Population," J. Neurotrauma, vol. 21, no. 10, pp. 1371-1383, 2004.

[2] R. J. Triolo, D. E. Robinson, and R. R. Betz, "Force-velocity and length-tension properties of stimulated human quadriceps muscle in spinal cord injured children," Proc. IEEE Conf. Eng. Med. Biol. Soc., vol. 3, pp. 967-968, 1989.

[3] M. J. Dolan, B. J. Andrews, and P. H. Veltink, "Switching Curve Controller for FES-Assisted Standing Up and Sitting Down," IEEE Trans. Rehab. Eng., vol. 6, no. 2, pp. 167-171, 1998.

[4] M. S. Poboroniuc, D. E. Wood, R. Riener, and N. N. Donaldson, "A New Controller for FES-Assisted Sitting Down in Paraplegia," Adv. Electrical and Comp. Eng., vol. 10, no. 4, pp. 9-16, 2010.

[5] "NASA-STD-3000 Man-Systems Integration Standards," vol. 1, section 3, 2000.

[6] S. Yoshioka, A. Nagano, R. Himeno, and S. Fukashiro, "Computation of the kinematics and the minimum peak joint moments of sit-tostand movements," Biomed. Eng. Online, vol. 6, no. 26, pp. 2007. 